\title{
Processo de adoecimento da criança indígena e suas implicações para a mortalidade infantil
}

Disease process of the indigenous child and its implications for infant mortality

Proceso de la enfermedad de los niños indígenas y sus implicaciones para la mortalidad infantil

Érica Baggio ${ }^{1}$; Taiana Aparecida Duarte Grein ${ }^{2}$; Rafael Fernandes Demarchi ${ }^{3}$; Michele de Melo Mariano ${ }^{4}$; Vagner Ferreira do Nascimento ${ }^{5}$; Thalise Yuri Hattori ${ }^{6}$; Ana Cláudia Pereira Terças ${ }^{7}$; Alisséia Guimarães Lemes ${ }^{8}$

\section{Resumo}

A criança indígena encontra-se atualmente num cenário de vulnerabilidade social, devido principalmente a sua condição histórica e social marcada de grandes transformações. A existência das desigualdades éticas e raciais deixa-as dependentes de um olhar diferenciado nos serviços de saúde, haja vista a forte discrepância nos fatores que contribuem para o adoecimento da criança indígena e seu índice de mortalidade infantil. Objetivou-se através desse estudo conhecer o processo de adoecimento da criança indígena brasileira e suas implicações para a mortalidade infantil. Tratase de uma revisão de literatura realizada no mês de dezembro de 2015, a partir de busca eletrônica na Biblioteca Virtual de Saúde (BVS), nas seguintes bases de dados Scielo, Medline e Lilacs, utilizando os descritores: população indígena, criança e cultura, com o operador booleano "and". Para seleção das publicações a serem incluídas na revisão adotou-se como critérios de inclusão: artigos, teses e documentos científicos, no idioma Português (Brasil), publicados entre os anos de 2005 a 2015. Obteve-se 14 materiais, sendo excluídos 4 que não se relacionavam com o tema. Os resultados apontam um processo de adoecimento indígena marcado pelas precárias condições de infraestrutura e acesso a informações de saúde. As doenças prevalentes entre esse público são as

\footnotetext{
${ }^{1}$ Graduanda em Enfermagem pela Universidade do Estado de Mato Grosso (UNEMAT). Departamento de Enfermagem - Campus Tangará da Serra. Rua Euclides Geraldo de Medeiros, 295 E, Centro, Tangará da Serra, Mato Grosso, Brasil. CEP: 78300-000. email: baggio.1994@ hotmail.com

${ }^{2}$ Graduanda em Enfermagem pela Universidade do Estado de Mato Grosso (UNEMAT). Departamento de Enfermagem - Campus Tangará da Serra. Rua 3, 488 N, Centro, Tangará da Serra, Mato Grosso, Brasil. CEP: 78300-000. email: taiana_grein@hotmail.com

${ }^{3}$ Graduando em Enfermagem pela Universidade do Estado de Mato Grosso (UNEMAT). Departamento de Enfermagem - Campus Tangará da Serra. Rua 24, Centro, Tangará da Serra, Mato Grosso, Brasil. CEP: 78300-000. email: rafael.demarchi@hotmail.com

${ }^{4}$ Graduanda em Enfermagem pela Universidade do Estado de Mato Grosso (UNEMAT). Departamento de Enfermagem - Campus Tangará da Serra. Rua José Cândido Melhorança, 263 E, Centro, Tangará da Serra, Mato Grosso, Brasil. CEP: 78300-000. email: michelemello@hotmail.co.uk

${ }^{5}$ Mestre em Terapia Intensiva pela Sociedade Brasileira de Terapia Intensiva. Docente Assistente da Universidade do Estado de Mato Grosso (UNEMAT). Departamento de Enfermagem - Campus Tangará da Serra. Rua Moreira Cabral, 475, Campinas, Barra do Garças, Mato Grosso, Brasil. CEP: 78600-000. E-mail: vagnerschon@ hotmail.com ${ }^{6}$ Mestre em Ciências da Saúde, Doenças Crônicas e Infecto-Parasitárias, pela Universidade Federal da Grande Dourados (UFGD). Docente Assistente da Universidade do Estado de Mato Grosso (UNEMAT). Departamento de Enfermagem - Campus Tangará da Serra. Rua 12 A, 1432 W, Jardim Itália, Tangará da Serra, Mato Grosso, Brasil. CEP: 78300-000. E-mail: thalisehattori@gmail.com

${ }^{7}$ Mestre em Saúde Coletiva, Epidemiologia, pela Universidade Federal de Mato Grosso (UFMT). Docente Assistente da Universidade do Estado de Mato Grosso (UNEMAT). Departamento de Enfermagem - Campus Tangará da Serra. Rua 32, 152 N, Centro, Tangará da Serra, Mato Grosso, Brasil. CEP: 78300-000. E-mail: enfanacnp@gmail.com ${ }^{8}$ Mestre em Imunologia e Parasitologia Básicas e Aplicadas, pela Universidade Federal de Mato Grosso (UFMT). Docente Assistente da Universidade Federal de Mato Grosso (UFMT). Departamento de Enfermagem - Campus Barra do Garças. Avenida Valdon Varjão, 6.390, Barra do Garças, Mato Grosso, Brasil. CEP: 78600-000. E-mail: alisseia@hotmail.com
} 
ISSN 2179-6750

infecciosas, parasitárias, e as carências nutricionais, a desnutrição e a anemia. Tais achados contribuem para a taxa de mortalidade entre as crianças indígenas, que permanece superior a das demais crianças, constituindo um importante problema de saúde pública.

Descritores: População Indígena; Criança; Cultura; Antropologia

\section{Abstract}

Indigenous child currently lies in a setting of social vulnerability, mainly due to its historical and social condition marked major changes. The existence of ethical and racial inequalities leaves them dependent on a different look in the health services, given the strong discrepancy in the factors that contribute to the illness of indigenous children and their infant mortality rate. This study aimed to know through this study the disease process of the Brazilian Indian child and its implications for infant mortality. This is a literature review conducted in December 2015, from electronic search in the Virtual Health Library (VHL), the following databases Scielo, Medline and Lilacs, using the descriptors: indigenous people, children and culture with the Boolean operator "and". For selection of the publications to be included in the review it is adopted as inclusion criteria: articles, theses and scientific papers, in Portuguese language (Brazil), published between the years 2005 to 2015 was obtained 14 materials were excluded 4 that They not related to the theme. The results show an indigenous disease process marked by poor infrastructure conditions and access to health information. The prevalent diseases among this audience are infectious, parasitic, and nutritional deficiencies, malnutrition and anemia. These findings contribute to the mortality rate among indigenous children, which remains more than the other children, constituting a major public health problem.

Key-words: Indigenous People; Child; Culture; Anthropology

\section{Resumen}

El niño indígena actualmente se encuentra en un entorno de vulnerabilidad social, principalmente debido a su condición histórica y social marcado cambios importantes. La existencia de desigualdades raciales éticos y los deja depende de un aspecto diferente en los servicios de salud, dada la fuerte discrepancia en los factores que contribuyen a la enfermedad de los niños indígenas y su tasa de mortalidad infantil. Este estudio tuvo como objetivo conocer a través de este estudio, el proceso de la enfermedad del niño indígena brasileña y sus implicaciones para la mortalidad infantil. Se trata de una revisión de la literatura realizada en diciembre de 2015, de la búsqueda electrónica en la Biblioteca Virtual en Salud (BVS), las siguientes bases de datos Scielo, Lilacs y Medline, utilizando los descriptores: los indígenas, los niños y la cultura con el operador booleano "y". Para la selección de las publicaciones que se incluirán en la revisión que se adopta como criterios de inclusión: artículos, tesis y artículos científicos, en idioma portugués (Brasil), publicados entre los años 2005 a 2015 representaba obtenido 14 materias que se excluyeron 4 ellos no están relacionados con el tema. Los resultados muestran un proceso de la enfermedad indígena marcada por las condiciones de infraestructura y pobres el acceso a la información sanitaria. Las enfermedades prevalentes en esta audiencia son deficiencias infecciosas, parasitarias y nutricionales, la malnutrición y la anemia. Estos resultados contribuyen a la tasa de mortalidad entre los niños indígenas, que sigue siendo más que los otros niños, lo que constituye un importante problema de salud pública.

Palabras-claves: Población Indígena; Niño; Cultura; Antropología 


\section{Introdução}

As mudanças ocorridas com o processo de urbanização em diferentes regiões do mundo têm gerado impacto em diferentes setores da sociedade e, por conseguinte, na saúde. Se por um lado houve melhoria nas condições de vida no Brasil, por outro, parte da população ainda vive em condições inadequadas de saúde, padecendo as desigualdades sociais. O resultado dessa disparidade ocasiona graves iniquidades, a exemplo da maior mortalidade infantil entre as crianças indígenas menores de cinco anos de idade se comparadas com as demais crianças dessa mesma faixa etária ${ }^{1,2}$.

A maior susceptibilidade da criança indígena, devido principalmente a sua condição histórica e social marcada de grandes transformações, sobretudo após a chegada da cultura ocidental, torna-as dependentes de um olhar diferenciado, de uma atenção especializada nos serviços de saúde, haja vista que doenças infectocontagiosas, precariedade nutricional, deficiência de acesso e cuidados com a saúde são problemas comuns que assolam esse grupo e muitas vezes são negligenciados por falta de conhecimento e habilidade no agir ${ }^{2,3}$.

A forte discrepância nos fatores que contribuem para o adoecimento da criança indígena e seu índice de mortalidade infantil são apontados no Relatório dos Objetivos de Desenvolvimento do Milênio (2013), desenvolvido pela Organização das Nações Unidas (ONU), onde mostra que, embora a mortalidade mundial tenha tido um decréscimo de $47 \%$ de óbitos por mil nascidos vivos, o nível de mortalidade ainda é elevado, preocupante e de causas heterogêneas nas crianças brasileiras, o que persiste nas grandes diferenças regionais e raciais ${ }^{1}$.

Nos últimos anos, algumas políticas voltadas à criança vêm sendo discutidas com o propósito de dar maior visibilidade a esse público, que por muitos anos, foram tratadas sem distinção dos adultos, sem o devido enfoque aos aspectos relacionados ao crescimento e desenvolvimento ${ }^{4,5}$. O Ministério da Saúde institui em 2015, a Política Nacional de Atenção Integral à Saúde da Criança (PNAISC) no âmbito do Sistema Único de Saúde (SUS) que prioriza à primeira infância bem como às populações de maior vulnerabilidade como as crianças com deficiências, indígenas, quilombolas e ribeirinhas, merecendo essas uma atenção especializada, ancorada no seu processo sociocultural ${ }^{6,1}$.

Entretanto, ainda se verifica nas crianças indígenas de muitas aldeias o desmame precoce, dificuldade de acesso aos serviços de saúde, hábitos alimentares e de higiene pessoais precários, além de condições ambientais desfavoráveis (convivência direta com animais domésticos, falta de infraestrutura adequada para o abastecimento de água potável, falta do hábito de lavar as mãos, ausência de coleta de lixo e despeja de dejetos nos ambiente vizinhos). Tal situação contribui para um cenário de disparidade étnica cultural, com aumento de doenças infectocontagiosas e 
ISSN 2179-6750

desequilíbrio nutricional, vivenciado por muitas etnias ${ }^{7,8}$.

Vislumbrando esse cenário de grandes diversidades étnicas e raciais, bem como de transformações socioculturais, objetivou-se com o estudo conhecer o processo de adoecimento da criança indígena brasileira e suas implicações para a mortalidade infantil, a partir de publicações científicas de algumas etnias brasileiras que foram estudadas.

\section{Métodos}

Trata-se de uma revisão de literatura, com característica descritiva. A pesquisa bibliográfica se caracteriza por utilizar fonte de dados secundários. Possui caráter exploratório e permite uma maior familiaridade com o assunto, aperfeiçoamento de ideias ou revelação de intuições. Já a pesquisa descritiva busca descrever características de grupos, conhecer processos e levantar opiniões, atitudes e crenças da população estudada, compreendendo a realidade cotidiana ${ }^{10}$.

Inicialmente, definiu-se a questão que norteou o estudo, que consistiu em: Como ocorre o processo de adoecimento da criança indígena brasileira e quais suas implicações para a mortalidade infantil, a partir de pesquisas científicas?

A coleta de dados ocorreu por meio de busca eletrônica na Biblioteca Virtual de Saúde (BVS), nas seguintes bases de dados Scielo, Medline e Lilacs, utilizando os descritores em ciências da saúde: população indígena, criança e cultura, com o operador booleano "and". Adotou-se como critérios de inclusão: artigos, teses e documentos científicos, na íntegra, no idioma Português (Brasil), publicados entre os anos de 2005 a 2015.

O levantamento dos dados foi realizado no mês de dezembro de 2015, obtendo 14 publicações. Inicialmente, os materiais foram selecionados por meio da leitura do título e do resumo avaliados independentemente por dois avaliadores. Aqueles que fossem aprovados pelos dois avaliadores foram incluídos no estudo. Os que apresentaram discordância foram submetidos a um terceiro avaliador. Destes, foram excluídos 4 que não se relacionavam com o tema ou que não contemplavam os critérios de inclusão. Assim, a amostra final desta revisão foi constituída por 10 publicações conforme apresentado em quadro sinóptico. Os demais artigos citados no texto foram utilizados para discussão dos achados.

Devido à heterogeneidade dos estudos, os dados foram agrupados e analisados de modo descritivo, de maneira a responder aos objetivos da pesquisa. Não houve conflito de interesses na condução desta revisão.

Pela pequena e limitada disponibilidade de estudos com crianças indígenas brasileiras, optou-se por não definir uma etnia específica, mesmo na compreensão de particularidades culturais. 
ISSN 2179-6750

Mas, a descoberta de características que definem a diferença de crianças indígenas para as não indígenas, viabilizou o desenvolvimento do estudo sob esse enfoque.

Foram respeitados todos os aspectos éticos em pesquisa com esse caráter documental, sinalizando e informando todas as fontes de dados utilizadas.

\section{Resultados e Discussão}

Os povos indígenas estão presentes em todas as regiões do Brasil. São mais de 200 povos, divididos entre 305 etnias, falantes de aproximadamente 180 línguas e dialetos, em territórios rurais e urbanos, com características sociais, culturais, assim como trajetórias históricas, econômicas e políticas distintas ${ }^{11,12}$. Tais características contribuem para a diversidade cultural existente nos povos indígenas, que repercutem em modos únicos de perceber a criança, os quais podem ou não contribuir para o aparecimento de enfermidades.

Alguns estudiosos, como Yamamotto ${ }^{13}$, explicam que os fatores causais para as afecções na cultura indígena estão classificadas basicamente em dois grupos: os místicos e os naturais. O primeiro se caracteriza pelo fato de os índios interpretarem que as doenças estão associadas a crenças religiosas e o sofrimento humano a domínios espirituais e alterações da alma. Já no segundo grupo, eles consideram fatores relacionados ao ambiente, como a chuva, a seca e a estação do ano, além de aspectos individuais, como estresse, desânimo e alimentação inadequada, como fatores que determinam o desequilíbrio do corpo. Com relação ao processo saúde-doença da criança indígena, muitas tribos acreditam que os pais são os responsáveis, sendo a patologia resultada de algum fator causal realizado ou cometido pelos genitores.

Alguns povos, por exemplo, os Kaiowá e Guarani se apresentam socialmente e estruturalmente através da parentela, também denominado família extensa, onde pessoas criam círculos de vínculos parenterais tendo como figura central, o líder. O cuidado à saúde e formação da criança Kaiowá e Guarani fica sob responsabilidade da parentela, que deverá identificar os problemas sociais e de saúde presente nesse público, e não de profissionais de saúde qualificados, colocados por órgãos externos ${ }^{14}$.

Tais órgãos, como a Fundação Nacional de Saúde (FUNASA), apesar de se mostrarem importantes no monitoramento de diversas situações de saúde como o pré-natal, vacinação e exames preventivos das crianças, por exemplo, tem sido avaliado por alguns estudiosos no que concerne ao sistema de saúde adotado para a comunidade indígena, que se mostra externo a realidade vivenciada por esses povos e incapaz de atuar em um contexto intercultural ${ }^{14}$. 


\section{ISSN 2179-6750}

Quadro 1. Quadro sinóptico com as principais características das publicações selecionadas.

\begin{tabular}{|c|c|c|c|}
\hline $\begin{array}{c}\text { Ano de } \\
\text { publicação }\end{array}$ & Autores & Objetivos & Principais conclusões \\
\hline 2009 & Ferreira $\mathrm{AA}^{17}$ & $\begin{array}{l}\text { Descrever o estado nutricional de } \\
\text { crianças indígenas Xavante de } \\
\text { Pimentel Barbosa/ Etênhiritipá } \\
\text { (MT) e analisar os fatores que estão } \\
\text { associados ao crescimento. }\end{array}$ & $\begin{array}{l}\text { Compreende as condições de vida e os } \\
\text { fatores que explicam, mesmo que } \\
\text { parcialmente, o estado nutricional das } \\
\text { crianças indígenas. }\end{array}$ \\
\hline 2011 & Fávaro TR ${ }^{20}$ & $\begin{array}{l}\text { Traçar o perfil nutricional do povo } \\
\text { Xukuru de Ororubá, na perspectiva } \\
\text { de situá-los no processo de } \\
\text { transformações nutricionais ao } \\
\text { longo dos anos no Brasil. }\end{array}$ & $\begin{array}{c}\text { O perfil nutricional dos Xukuru apresenta } \\
\text { semelhanças com o processo de transição } \\
\text { nutricional da população brasileira não } \\
\text { indígena, com presença de anemia em } \\
\text { crianças e baixa prevalência de desnutrição } \\
\text { infantil. }\end{array}$ \\
\hline 2011 & Barreto $\mathrm{CTG}^{21}$ & $\begin{array}{c}\text { Caracterizar o estado nutricional } \\
\text { das crianças indígenas Guarani < } 5 \\
\text { anos residente em } 5 \text { aldeias do } \\
\text { litoral da região sudeste do Brasil. }\end{array}$ & $\begin{array}{l}\text { Foram evidenciadas elevadas prevalências } \\
\text { de desnutrição e anemia entre as crianças } \\
\text { Guarani }<5 \text { anos. Esses resultados, aliados à } \\
\text { relevância epidemiológica das IRA no } \\
\text { grupo, reforça a hipótese de perpetuação de } \\
\text { um círculo } \\
\text { vicioso desnutrição-infecção. }\end{array}$ \\
\hline 2011 & $\begin{array}{c}\text { Ferreira MEV, } \\
\text { Matsuo T, Souza } \\
\text { RKT }^{24}\end{array}$ & $\begin{array}{l}\text { Analisar os aspectos demográficos e } \\
\text { o padrão de mortalidade das } \\
\text { populações indígenas aldeada no } \\
\text { Estado do Mato Grosso do Sul, } \\
\text { Brasil, comparativamente ao da } \\
\text { população total do estado, no intuito } \\
\text { de aprimorar os conhecimentos } \\
\text { acerca das condições de saúde das } \\
\text { populações indígenas }\end{array}$ & $\begin{array}{l}\text { As taxas mais elevadas de mortalidade em } \\
\text { idades precoces são por doenças infecciosas } \\
\text { e parasitárias. Conclui-se que as condições } \\
\text { de saúde da população indígena são piores } \\
\text { que a da população total. }\end{array}$ \\
\hline 2013 & $\begin{array}{l}\text { Assis EM, Olivieria } \\
\text { RC, Moreira LE, } \\
\text { Pena JL, Rodrigues } \\
\text { LC, Machado-Coelho } \\
\text { GLL }^{9}\end{array}$ & $\begin{array}{c}\text { Determinar a prevalência de } \\
\text { parasitos intestinais na etnia } \\
\text { Maxakali em Minas Gerais, Brasil. }\end{array}$ & $\begin{array}{l}\text { A prevalência de parasitos }(89,5 \%) \text { e do } \\
\text { poliparasitismo (46\%) foi semelhante } \\
\text { quanto ao sexo e idade. Pode-se concluir } \\
\text { que esses povos vivem em vulnerabilidade } \\
\text { social. }\end{array}$ \\
\hline 2013 & $\begin{array}{l}\text { Belo EN, Orellana } \\
\text { JDY, Levino A, Basta } \\
\text { PC }^{22}\end{array}$ & $\begin{array}{c}\text { Descrever a situação } \\
\text { epidemiológica da tuberculose, } \\
\text { mapear a sua incidência e investigar } \\
\text { fatores associados ao abandono do } \\
\text { tratamento nos municípios do } \\
\text { Amazonas, Brasil. }\end{array}$ & $\begin{array}{l}\text { Predomina elevadas taxas de incidência por } \\
\text { tuberculose em indígenas. }\end{array}$ \\
\hline 2013 & Coelho CVL ${ }^{18}$ & $\begin{array}{l}\text { Investigar marcadores genotípicos e } \\
\text { fenotípicos em amostras de } E \text {. coli } \\
\text { isoladas de crianças de diferentes } \\
\text { aldeias Guarani, Rio de Janeiro. }\end{array}$ & $\begin{array}{c}\text { Detecção de patotipos intestinais e } \\
\text { circulação de amostras multirresistentes, } \\
\text { que alerta para ações específicas na área de } \\
\text { vigilância em saúde indígena. }\end{array}$ \\
\hline 2014 & Santos LP ${ }^{19}$ & $\begin{array}{l}\text { Analisar a efetividade das vacinas } \\
\text { presentes no calendário vacinal } \\
\text { indígena (2007-2008) } \\
\text { na prevenção da hospitalização por } \\
\text { DRAB. }\end{array}$ & $\begin{array}{l}\text { São necessários mais estudos sobre vacinas } \\
\text { com populações indígenas, buscando } \\
\text { superar algumas limitações encontradas, } \\
\text { que poderia justificar a ausência de } \\
\text { efetividade das vacinas neste estudo. }\end{array}$ \\
\hline 2014 & $\begin{array}{l}\text { Silva OLO, } \\
\text { Lindemann IL, Prado } \\
\text { SG, Freitas KC, } \\
\text { Souza AS }{ }^{28}\end{array}$ & $\begin{array}{l}\text { Descrever a evolução do Sistema de } \\
\text { Vigilância Alimentar e Nutricional } \\
\text { (Sisvan) e do estado nutricional de } \\
\text { crianças indígenas ( }<5 \text { anos) no } \\
\text { estado de Mato Grosso do Sul, } \\
\text { Brasil, no período de } 2002 \text { a } 2011 .\end{array}$ & $\begin{array}{c}\text { Aumentou a cobertura do Sisvan indígena } \\
\text { (de } 82,0 \% \text { em } 2002 \text { para } 97,2 \% \text { em 2011) e } \\
\text { reduziu a desnutrição infantil ( de } 16,0 \% \\
\text { para 6,5\%) no período estudado, porém } \\
\text { não se pode negligenciar os grandes } \\
\text { desafios sobre essas temáticas nesses } \\
\text { povos. }\end{array}$ \\
\hline 2014 & $\begin{array}{l}\text { Tagliari IA, Barros } \\
\text { Filho AA, Rocha } \\
\text { Ferreira MB }\end{array}$ & $\begin{array}{l}\text { Comparar a performance motora em } \\
\text { escolares de } 8 \text { e } 9 \text { anos de idade, } \\
\text { residentes em Terra Indígena, em } \\
\text { zona urbana e zona rural do } \\
\text { Município de Nova Laranjeiras, } \\
\text { Estado do Paraná, Brasil. }\end{array}$ & $\begin{array}{l}\text { Nos testes de performance motora, } \\
\text { controlados pela idade, peso e estatura, } \\
\text { ocorrem diferenças significativas no teste } \\
\text { de flexibilidade, favorecendo as crianças } \\
\text { indígenas, já o desenvolvimento da força e } \\
\text { da resistência abdominal das crianças } \\
\text { indígenas necessita de maior atenção, a fim } \\
\text { de evitar desvios posturais e algia lombar. }\end{array}$ \\
\hline
\end{tabular}

Fonte: Elaborado pelos próprios autores. 
ISSN 2179-6750

Embora reconheçam que os saberes e recursos técnicos da medicina não indígena não poderão substituir a tradicional, ocorre uma desvalorização dos rituais culturais e religiosos dos indígenas, podendo ser verificados através da diminuição parcial ou em alguns casos total de partos realizados por parteiras, sendo as gestantes encaminhadas cada vez mais cedo à unidade hospitalar e submetidas ao parto cesária, prejudicando a aproximação da índia parturiente de sua família e comunidade. Também se observa cada vez menos presente o benzedor, o xamã, o curandeiro e a medicina natural, elevando-se o uso e abuso da medicação industrial ${ }^{15}$.

Os resultados de pesquisas, observados no quadro sinóptico, incitam que as doenças que mais prevalecem nas crianças indígenas são as doenças parasitárias, Infecções Respiratórias Agudas (IRAS) e com um aumento significativo nos últimos anos às relacionadas ao estado nutricional, como a anemia ${ }^{16-22}$.

Conforme o estudo de Coelho ${ }^{18}$, demonstrado no quadro sinóptico, as doenças infecciosas e parasitárias, dentre elas, as gastroenterites, são causadas por parasitas que ao se alojar no hospedeiro desencadeiam quadros infecciosos, na maioria das vezes com manifestações diarreicas. Elas estão presentes em áreas com precárias condições de saneamento e representam um grave problema de saúde pública. Esse mesmo autor ressalta que na população indígena elas estão bem disseminadas e ocorrem com maior frequência, sendo uma das causa de morbimortalidade infantis mais significativas, senão a mais importante.

Assis et al ${ }^{9}$ encontrou em sua pesquisa uma prevalência semelhante, se comparado sexo e idade, de parasitos $(89,5 \%)$ e poliparasitismo (46\%) na etnia Maxakali em Minas Gerais. Esse autor afirma que tais resultados, de elevados níveis de parasitismo intestinais entre os Maxakali, indicam precárias condições de saneamento entre esses povos, colocando-os em situações de vulnerabilidade social. Dado ao exposto, medidas de infraestrutura e educação em saúde precisam ser implementadas urgentemente pelas autoridades responsáveis, destacando-se o papel da atenção primária a saúde como base desse cuidado e intervenção. Entretanto, os desafios enfrentados pelos indígenas não se limitam apenas às doenças e ausência de saneamento, mas necessita de um olhar mais amplo e abrangente, a começar pela criação de políticas públicas que respeitem e priorizem seus aspectos socioculturais.

Em estudo realizado por Ferraz ${ }^{23}$ junto a duas equipes da Estratégia Saúde da Família que atuam na assistência á saúde dos povos Kaingang e Guarani em dois municípios do estado de Santa Catarina - Brasil encontrou-se alguns hábitos da própria cultura indígena que podem contribuir para esse adoecimento. Segundo os informantes, à medida que as crianças crescem, são dadas a essas a responsabilidade e autonomia do autocuidado, principalmente com relação à alimentação, higiene e 
ISSN 2179-6750

conforto. É como se a partir de então tivessem que "se virar sozinhas" mesmo estando em uma faixa-etária que necessite de acompanhamento e cuidados. Verifica-se nesse ponto o papel crucial da equipe multiprofissional na coordenação das informações do cuidado.

Estudo realizado por Ferreira, Matsuo e Souza ${ }^{24}$ com a população Xavante, no estado de Mato Grosso, pode-se evidenciar na aldeia a falta de coleta de lixo e destino inadequado de dejetos, que também contribui para a circulação de parasitoses infantis. A água utilizada até para beber, era proveniente de córregos, sem tratamento, onde os mesmos lavavam roupas e utensílios e às vezes evacuavam nas proximidades, sem considerar ainda que as crianças ao andarem descalças ficavam mais susceptíveis ao contato com as fezes. Tais fatores podem ser agravados ao considerar à restrição territorial indígena, ficando cada vez mais próximos da comunidade e cultura do homem branco, limitando seu espaço de convívio ${ }^{25,26}$.

Além das informações referentes ao cuidado com a saúde, a equipe da saúde da família pode atuar de diferentes maneiras a intervir no desarranjo biopsicossocial. Conforme destacado por Santos ${ }^{19}$ a atualização do calendário vacinal indígena é uma estratégia que contribui para reduzir muitas doenças, principalmente na infância, dentre elas as doenças respiratórias agudas baixas (DRAB). Reflete também em uma situação mais geral no cuidado e acesso a saúde, e tem extrema importante na redução da morbimortalidade por doenças comuns nesse público.

Embora sejam necessários mais estudos sobre vacinas com populações indígenas, buscando superar algumas limitações encontradas no estudo de Santos ${ }^{19}$ com crianças Guarani, onde as vacinas não tiveram muita efetividade, ressalta-se que a adequação do esquema vacinal preconizado e a qualidade dos imunobiológicos ofertados geram subsídios para a prevenção de doenças imunopreveníveis. Tal atitude também pode contribuir na diminuição das taxas de incidência de tuberculose encontrada no estudo de Belo ${ }^{22}$ com população amazonense da fronteira BrasilColômbia-Peru-Venezuela. Não poderá se descartar a dificuldade de acesso desses povos a saúde como justificativa para a alta taxa de incidência de tuberculose e abandono do tratamento.

Além de algumas condutas da própria cultura contribuir para as moléstias, os povos indígenas são considerados atualmente uma população de grande vulnerabilidade social, como mencionado anteriormente. Com a chegada da cultura do não índio, muitos hábitos comportamentais foram rendidos ao modo dominante e injusto da cultura ocidental, com resultados de perdas e desequilíbrios de muitos hábitos saudáveis ${ }^{27}$. Nas últimas décadas, observa-se uma maior vulnerabilidade da população indígena em relação aos avanços dos demais povos brasileiros, principalmente no que diz respeito à saúde, saneamento, habitação e educação ${ }^{7}$.

Barreto ${ }^{21}$, Ferreira ${ }^{17}$ e Silva et al ${ }^{28}$ apontam alguns fatores que podem contribuir para essas 141 
ISSN 2179-6750

causas, tais como acesso limitado às ações de atenção primária nas aldeias, pois, muitas ainda vivem em áreas distantes o que dificultam o acesso à assistência e ao serviço de saúde, condições socioeconômicas precárias, desigualdade sanitária e ambiental, que interferem no ser e viver da criança indígena.

Observa-se que a baixa cobertura assistencial e educativa para à saúde, o que de outra maneira poderia contribuir para evitar muitos adoecimentos, inclusive àqueles ligados ao pré-natal por parte das gestantes indígenas e puericultura das crianças menores de dois anos, com temáticas referentes ao aleitamento materno, alimentação complementar, cuidados com o recém-nascido, mudanças do processo gravídico-puerperal, contribui para o aparecimento de moléstias ${ }^{29,30}$.

Com a urbanização, a escassez de recursos naturais e modificação do modo de subsistência, observou-se a transição ao qual passaram ao adotar hábitos de vida das regiões urbanizadas. Com substituição dos antigos costumes de vida saudáveis da sua cultura, como o consumo de alimentos naturais, por alimentos industrializados, ocasionou impacto diretamente no crescimento e desenvolvimento das crianças, tendo várias repercussões em seu perfil nutricional ${ }^{27,31}$. Conforme observado no quadro sinóptico, estudos de Fávaro ${ }^{20}$, Barreto ${ }^{21}$ e Silva ${ }^{28}$, demonstraram que a obesidade e a anemia tem se destacado nas crianças indígenas de algumas aldeias, principalmente nas mais próximas da área urbana.

Todos esses fatores, além de contribuírem para as doenças prevalentes na infância, têm sido evidenciados como as principais causas de mortalidade infantil. As IRAs e as doenças parasitárias tem destacaram-se durante muitos anos e ainda continuam prevalentes, embora alguns estudos, como os realizados por Fávaro ${ }^{20}$ e Silva ${ }^{28}$ apontam que houve uma queda na prevalência de desnutrição infantil, com aumento moderado na prevalência de excesso de peso e elevada prevalência de anemia. Portanto, pode-se observar que o perfil nutricional desse público vem sendo modificado a partir da inserção da cultura do homem branco e também tem influenciado nos índices de mortalidade, uma vez que ocorreu uma transição nutricional com esses povos.

O I Inquérito Nacional de Saúde e Nutrição dos Povos Indígenas, realizado em 2008/2009, foi o primeiro estudo nacional de maior abrangência a relatar o perfil nutricional desses indivíduos (mulheres e crianças). Ocorreram muitas transformações demográficas, socioeconômicas, culturais, ambientais e no perfil epidemiológico nos últimos anos, que modificaram significativamente a dinâmica das comunidades indígenas. A substituição da caça, coleta e pesca decorrente de novos poderes econômicos atrelado à diminuição territorial para plantio, levou ao empobrecimento da dieta $^{32}$. Somado a isso, observa-se o aumento do consumo de açúcar, refrigerante, sal, entre outros produtos industrializados e a diminuição das atividades que demandam energia ${ }^{26,31}$. 
Dos estudos encontrados por Lício ${ }^{33}$ em periódicos nacionais e internacionais, a maioria demonstraram déficits antropométricos graves em crianças indígenas, especialmente com relação ao índice de estatura-para-idade (E/I). Nos Kaigang, considerado o quinto grupo mais populoso, o déficit de estatura para a idade variou entre $15 \%$ a $35 \%{ }^{35}$ e nos Kaiowás e Guaranis cerca de 35\%, em 137 crianças aleatoriamente avaliadas ${ }^{14}$.

O estado nutricional comprometido associado às condições de vida e hábitos inadequados afeta o processo de crescimento e desenvolvimento da criança, contribuem para o aparecimento das doenças prevalentes, que favorecem para a piora nutricional, o que consequentemente, reflete para o crescente número de óbitos ${ }^{20}$.

Estudo realizado por Tagliari ${ }^{34}$ no estado do Paraná quanto a performance postural de crianças indígenas demonstrou que crianças indígenas apresentam uma força e resistência abdominal inferior a das crianças não indígenas, e que embora seja um achado isolado nessa pesquisa, não poder-se-á descartar a possibilidade de estar associada ao estado nutricional, visto que muitos dos comprometimentos posturais estão relacionados a alimentação e inatividade física.

Algumas famílias indígenas, como a Tremembé, são beneficiadas com auxílio alimentação após diagnóstico de pobreza e desnutrição em crianças menores de cinco anos, e para aquelas que são amamentadas exclusivamente até seis meses, recebem ainda, a alimentação complementar como forma preventiva. Por se tratar de um grupo com baixas condições socioeconômicas, com desnutrição e mortalidade infantil acentuada, esse auxílio contribui para a melhoria na qualidade de vida e prevenção de desnutrição e anemia ${ }^{35}$.

Entre indígenas Xakriabá, a média de aleitamento materno exclusivo são inferiores as observadas na criança em geral, e águas, chás e outros líquidos são precocemente introduzidos, pois a crença transmitida pelos mais velhos é que saciam a sede, curam cólicas e complementa o leite materno ${ }^{36}$.

São essas atitudes que privam as crianças de receberem benefícios nutricionais, imunológicos e funcionais do leite materno de forma precoce, que podem contribuir para os agravos a saúde na infância e até mesmo na fase adulta ${ }^{36}$. Portanto precisam-se conhecer através de estudos as situações de saúde e adoecimento dessa população, mesmo que sendo de algumas etnias, a fim de intervir de maneira a transformar esse cenário obscuro.

\section{Considerações finais}

O cenário atual da saúde dos povos indígenas resulta da trajetória histórica, marcada por contrariedades para os indígenas em relação aos avanços sociais identificados no Brasil nas últimas 
ISSN 2179-6750

décadas, principalmente no campo da saúde, habitação, saneamento e educação. A chegada da cultura do homem branco trouxe hábitos comportamentais divergentes e, com o aumento da acessibilidade para as tecnologias e mídias ampliaram o consumo de produtos distintos dos tradicionais, alavancando o processo de adoecimento social e de saúde dessa população. Além disso, pode-se verificar a prevalência de algumas crenças, passadas de geração para geração, que não condizem com hábitos de saúde benéficos.

A taxa de mortalidade entre as crianças indígenas estão relacionados às precárias condições de saneamento, habitação e acesso a saúde, o que nas crianças em geral é visto como declínio nos últimos anos. Já as doenças que mais prevalecem entre esse público são as doenças infecciosas e parasitárias, as IRAs, acompanhadas da desnutrição e alta prevalência de anemia. Vale ressaltar que a desnutrição tem apresentado uma queda nos últimos estudos, aumentando os casos de obesidade.

A falta de educação em saúde e acesso aos serviços tem gerados prejuízos tanto na promoção da saúde como na prevenção e recuperação de doenças. Hábitos de higiene e infraestrutura inadequados, aleitamento materno exclusivo inferior ao período recomendado pelo Ministério da Saúde, deficiência de vacinação eficaz e interrupções de tratamentos contribui para a prevalência de doenças que levam para a mortalidade infantil desse público.

Esses achados indicam a necessidade de repensar as estratégias para convívio com esses povos, de modo que essa relação se estabeleça harmoniosamente, que não modifique a forma de vivência e convivência desses indivíduos. Ao mesmo tempo oferecer recursos para melhoria de suas condições de vida, com implementação de medidas de saneamento básico, educação e assistência periódica a saúde.

A criação e execução de políticas, programas e sistemas que controlem com mais qualidade e fidedignidade os dados referentes à saúde e mortalidade infantil na atenção primária a saúde, podem facilitar a compreensão desse cenário peculiar que auxiliará na manutenção do patrimônio biológico, social e cultural indígena. Também torna-se necessário o investimento na formação e qualificação dos profissionais que irão assistir esses povos em diferentes momentos da vida.

\section{Referências}

1. Brasil. Ministério da Saúde. Objetivos de Desenvolvimento do Milênio: Relatório Nacional de Acompanhamento [Internet]. Brasília: Ipea. 2014 [acesso em 2015 dez. 9]. Disponível em: http://www.pnud.org.br/Docs/5_RelatorioNacionalAcompanhamentoODM.pdf.

2. Cardoso AM, Coimbra Jr CEA, Barreto CTG, Werneck GL, Santos RV. Mortality among Guarani Indians in Southeastern and Southern Brazil. Cad Saude Publica. 2011; 27 Suppl 2: 144 
222-36.

3. Schmidt MI, Duncan BB, Silva GA, Menezes AM, Monteiro CA, Barreto SM, et al. Chronic non-communicable diseases in Brazil: burden and current challenges. Lancet 2011; 377:1949-61.

4. Ariés P. História social da criança e da família. 2.ed. Rio de Janeiro: Livros Técnicos e Científicos (LTC); 1981.

5. Frias PG, Mullachery PH, Giugliani ERJ. Políticas de saúde direcionadas às crianças brasileiras: breve histórico com enfoque na oferta de serviço de saúde. In: Saúde Brasil 2008: 20 anos de Sistema Único de Saúde (SUS) no Brasil [Internet]. Brasília: Ministério da Saúde; 2009 [acesso em 2016 jan. 9]. Disponível em: http://bvsms.saude.gov.br/bvs/publicacoes/saude_brasil_2008.pdf.

6. Brasil. Ministério da Saúde. Portaria $n^{\circ} 1.130$, de 5 de agosto de 2015. Institui a Política Nacional de Atenção Integral à Saúde da Criança (PNAISC) no âmbito do Sistema Único de Saúde (SUS). Diário Oficial [da] República Federativa do Brasil. 2015 ago. 6; Seção 1. p. 37.

7. Coimbra Júnior CEAC. Saúde e povos indígenas no Brasil: reflexões a partir do I Inquérito Nacional de Saúde e Nutrição Indígena. Cad Saúde Pública. 2014; 30(4): 855-9.

8. Ferreira AA, Welch JR, Santos RV, Gugelmin SA. Nutritional status and growth of indigenous Xavante children, Central Brazil. Nutrition Journal. 2012; 11(3): 1-9.

9. Assis EM, Oliveira RC, Moreira LE, Pena JL, Rodrigues LC, Coelho GLLM. Prevalência de parasitos intestinais na comunidade indígena Maxakali, Minas Gerais, Brasil, 2009. Cad Saúde Pública. 2013; 29(4): 681-90.

10. Gil A. Como elaborar projetos de pesquisa. São Paulo: Atlas; 2007.

11. Silva E. Povos Indígenas: História, Culturas e o Ensino a partir da Lei 11.645. Rev Historien UPE/Petrolina. 2012; 7: 39-49.

12. Pagliaro H. A revolução demográfica dos povos indígenas no Brasil: a experiência dos Kayabí do Parque Indígena do Xingu, Mato Grosso, Brasil, 1970-2007. Cad Saúde Pública. 2010; 26(3): 579-90.

13. Yamamoto RM. Medicina ocidental e medicina Indígena: a favor da criança indígena brasileira. In: Manual de atenção à saúde da criança indígena. Brasília: Funasa; 2004.

14. Pícoli RP. Saúde, doença e morte de crianças: um olhar segundo a percepção dos Kaiowá e Guarani [tese] [Internet]. São Paulo: Universidade de São Paulo; 2008. [acesso em 2016 jan. 20]. 228f. Disponível em: http://www.teses.usp.br/teses/disponiveis/6/6136/tde-05032009- 
ISSN 2179-6750

$141736 /$.

15. Rissardo LK, Moliterno ACM, Borghi AC, Carreira L. Práticas de cuidado ao recémnascido: percepção de famílias Kaingang. Cienc Cuid Saude. 2011; 10(4): 634-41.

16. Cardoso AM, Coimbra Júnior CEA, Tavares FG. Morbidade hospitalar indígena Guarani no Sul e Sudeste do Brasil. Rev Bras Epidemiol. 2010; 13(1): 21-34.

17. Ferreira AA. Estado nutricional e fatores associados ao crescimento de crianças indígenas Xavante, Mato Grosso [dissertação]. Rio de Janeiro: Escola Nacional de Saúde Pública Sergio Arouca; 2009. 133f.

18. Coelho CVL. Marcadores de enteropatogenicidade em amostras de Encherichia coli isoladas de crianças indígenas - etnia Guarani, Sul do estado do Rio de Janeiro [dissertação] [Internet]. Rio de Janeiro: Fundação Oswaldo Cruz; 2013. [acesso em 2016 jan. 25]. Disponível em: http://pesquisa.bvs.br/brasil/resource/pt/lil-681315.

19. Santos LP. Efetividade de vacinas contra a hospitalização por doença respiratória aguda baixa em crianças indígenas guarani menores de cinco anos no Sul e Sudeste do Brasil [dissertação] [Internet]. Rio de Janeiro: Fundação Oswaldo Cruz; 2014. [acesso em 2015 nov. 10]. Disponível em: http://bvssp.icict.fiocruz.br/lildbi/docsonline/get.php?id=4041.

20. Fávaro TR. Perfil nutricional da população indígena Xukuru de Ororubá, Pernambuco, Brasil [tese] [Internet]. Rio de Janeiro: Fundação Oswaldo Cruz; 2011. [acesso em 2015 nov. 10]. Disponível em: http://indiosnonordeste.com.br/wpcontent/uploads/2012 /08/Favaro-TR.-TeseFinal.pdf.

21. Barreto CTG. Estado nutricional de crianças indígenas guarani no sudeste do Brasil [dissertação]. Rio de Janeiro: Escola Nacional de Saúde Pública Sergio Arouca; 2011. 100f.

22. Belo EM, Orellana JDY, Levino A, Basta PC. Tuberculose nos municípios amazonenses da fronteira Brasil-Colômbia-Peru-Venezuela: situação epidemiológica e fatores associados ao abandono. Rev Panam Salud Publica. 2013; 34(5), 321-9.

23. Ferraz L. O cuidado prestado à criança pela família e a comunidade indígena na percepção dos profissionais da saúde [dissertação] [Internet]. Florianópolis: Universidade Federal de Santa Catarina; 2010. [acesso em 2015 nov. 25]. Disponível em: https://repositorio.ufsc.br/handle/123456789/93936.

24. Ferreira MEV, Matsuo T, Souza RKT. Aspectos demográficos e mortalidade de populações indígenas do Estado do Mato Grosso do Sul, Brasil. Cad Saúde Pública. 2011; 27(12): 23272339.

25. Souza LG, Santos RV, Coimbra Jr. CEA. Estrutura etária, natalidade e mortalidade do povo 146 
ISSN 2179-6750

indígena Xavante de Mato Grosso, Amazônia, Brasil. Ciênc Saúde Coletiva. 2010; 15 Suppl 1: $1465-73$.

26. Leite MS, Santos RV, Gugelmin SA, Coimbra Júnior CEA. Crescimento físico e perfil nutricional da população indígena Xavante de Sangradouro-Volta Grande, Mato Grosso, Brasil. Cad Saúde Pública. 2006; 22(2): 265-76.

27. Dias Júnior NM, Verona APA, Pena JL, Machado-Coelho GLL. Desigualdades demográficas e socioeconômicas entre brancos e indígenas no Brasil. REDES. 2010; 15(2): 50-65.

28. Silva OLO, Lindemann IL, Prado SG, Freitas KC, Souza AS. Vigilância alimentar e nutricional de crianças indígenas menores de cinco anos em Mato Grosso do Sul, 20022011. Epidemiol. Serv. Saúde, Brasília. 2014; 23(3): 541-6.

29. Rios L, Cutolo SA, Giatti LL, Castro M, Rocha AA, Toledo RF, Pelicioni MCF, Barreira LP, Santos JG. Prevalência de parasitos intestinais e aspectos socioambientais em comunidade indígena no distrito de Iauaretê, município de São Gabriel da Cachoeira (AM), Brasil. Saúde Soc. 2007; 16(2): 76-86.

30. Associação Brasileira de Saúde Coletiva. I Inquérito Nacional de Saúde e Nutrição dos Povos Indígenas. Relatório final, nº 7 . Rio de Janeiro: Funasa; 2009.

31. Lourenço AE, Santos RV, Orellana JDY, Coimbra Júnior CEA. Nutrition transition in Amazonian: obesity and socioeconomic change in the Suruí Indians from Brazil. Am J Hum Biol. 2008; 20(5): 564-571.

32. Santos RV, Coimbra Júnior CEA. Cenário e tendências da saúde e da epidemiologia dos povos indígenas do Brasil. In: Coimbra Júnior CEA, Santos RV, Escobar AL. Epidemiologia e saúde dos povos indígenas no Brasil. Rio de Janeiro: Fiocruz/ABRASCO; 2003.

33. Licio JSA. Estado nutricional de crianças indígenas no Brasil: uma revisão sistemática da literatura científica [dissertação] [Internet]. Rio de Janeiro: Fundação Oswaldo Cruz; 2009. [acesso em 2016 jan. 25]. Disponível em: http://www.arca.fiocruz.br/handle/icict/2532.

34. Tagliari IA, Barros Filho AA, Rocha Ferreira MB. Performance motora em crianças de terra indígena, de zonas urbana e rural. R. Bras. Ci. e Mov. 2014; 22(2): 98-104.

35. Nascimento FF, Oliveira EM, Nunes JE, Gubert FA, Ximenes Neto FRX, Vieiram NFC. Cuidado à saúde da comunidade indígena Tremembé: olhar dos profissionais de saúde. Saúde Coletiva. 2011; 8(51): 138-43.

36. Sírio MAO, Freitas SN, Figueiredo AM, Gouvêa GDR, Pena JL, Machado-Coelho GLL. Tempo de aleitamento materno entre indígenas Xakriabá aldeados em Minas Gerais, Sudeste do Brasil. Rev Nutr. 2015; 28(3): 241-252. 